\title{
Structure Analysis of a Hyper-Complex Approximant to Icosahedral Quasicrystal using 3D Electron Diffraction Tomography
}

\author{
Peter Oleynikov ${ }^{1}$, Yanhang $\mathrm{Ma}^{1}$, Nobuhisa Fujita ${ }^{2}$, Javier Garcia-Garcia ${ }^{3}$, Kyung Byung Yoon ${ }^{4}$, An \\ Pang Tsai ${ }^{2}$ and Osamu Terasaki ${ }^{1,5}$. \\ 1. Materials and Environmental Chemistry, Stockholm University, Stockholm, Sweden. \\ 2. IMRAM, Tohoku University, Sendai, Japan. \\ 3. Facultad CC. Químicas, Universidad Complutense de Madrid, Madrid, Spain. \\ 4. Department of Chemistry, Sogang University, Seoul, Republic of Korea. \\ 5. Graduate School of EEWS, KAIST, Daejeong, Republic of Korea.
}

Analyzing the crystal structure of approximants is of vital importance in deriving structural information of building units (or basic clusters) and their arrangements toward icosahedral quasicrystals (IQCs). The acquired knowledge is essential in performing a hyper-space modeling, which is the only feasible way of today to elucidate the structural details of IQC's. Since approximants are periodic crystals, conventional single-crystal X-ray diffraction can in principle be applied to analyze their atomic structure. However, the task becomes quite challenging when it comes to the case of approximants to Al-based F-type IQCs, Al-Pd-TM (TM = transition metal) [1]. These approximants often have very large unit cells with lattice constants of over a few tens of Angströms [2]. A recent study also suggests that, except for the solved case of [2], it is often very difficult to grow single crystals having coherent crystallinity within the width of the incident X-ray beam. It is therefore desirable if the crystal structure can be assessed using electron diffraction from a sub-micron sized crystal domain.

The aim of this study is to assess the possibility of taking the advantage of 3D Electron Diffraction Tomography (3D EDT) [3] in order to solve the crystal structure of the Al-Pd-TM IQC approximant (cubic, s.g. $\mathrm{Pa}-3, \mathrm{a}=40.54 \AA$ ). Automated $3 \mathrm{D}$ EDT is a fast and efficient technique that has been recently developed by us [3]. It can be used for fast 3D reciprocal space scanning with a given fine step $\left(0.01^{\circ}-0.1^{\circ}\right)$ using conventional transmission electron microscopes.

The crystal structure of the individual sub-micron single crystal was determined from the EDT data collected in conventional selected area electron diffraction (SAED) mode using EDT-COLLECT software package [3] on JEOL JEM-2100 FEG CTEM equipped with a single high tilt holder $\left(+/-50^{\circ}\right)$ and Gatan UltraScan 1000 CCD (2048*2048). The acquired data set contains $\sim 2000$ unique electron diffraction patterns (exposure $0.5 \mathrm{sec} /$ frame). Reciprocal space coverage was $\sim 90^{\circ}$. The recorded frames were processed using the EDT-PROCESS software package [3] and assembled into a corresponding 3D volumetric representation of reciprocal space (Fig. 1). The crystal structure was successfully determined (Fig. 2) using the direct methods software Sir2011 [4] from the integrated intensities extracted by EDTPROCESS program.

In this work we show that 3D EDT as a very powerful technique which offers a facile and systematic way to study complex crystal structures. The authors would like to thank the following people and organizations [5]. 


\section{References:}

[1] A.P. Tsai et al, Mater. Trans. JIM 31 (1990), pp. 98-103.

[2] N. Fujita et al, Acta Cryst. A, 69 (2013), pp. 322-340.

[3] M. Gemmi and P. Oleynikov, Z. Kristallogr. 228 (2013), pp. 51-58.

[4] M.C. Burla et al, J. Appl. Cryst. 45 (2012), pp. 357-361.

[5] We kindly acknowledge Swedish Research Council (VR, 1486801), JEOL Ltd., Japan, Knut and Alice Wallenberg Foundation (3DEM-NATUR) and BK21Plus, Republic of Korea.

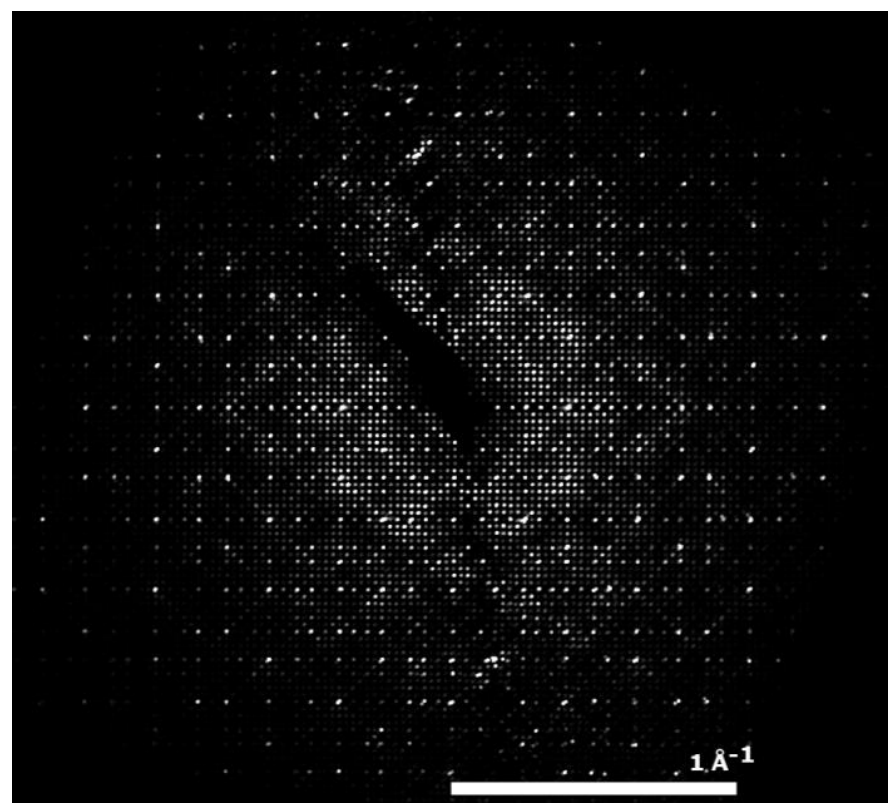

Figure 1. Reconstructed 3D reciprocal space along 111 direction.

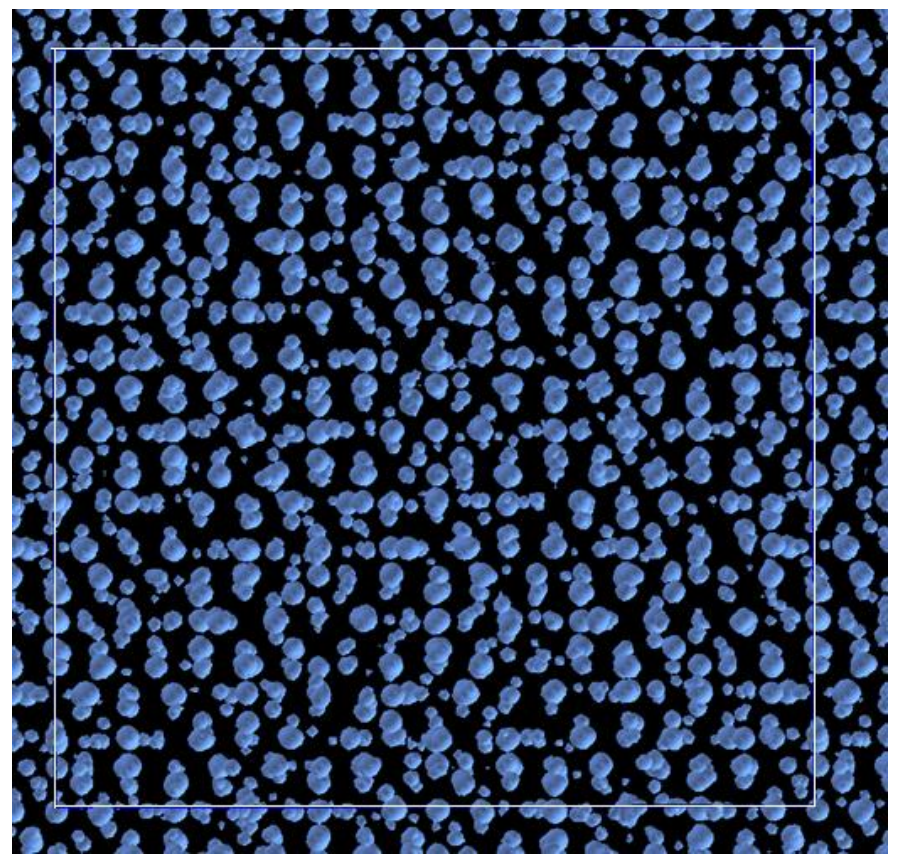

Figure 2. The potential map of the solved structure using direct methods. 